Urologe 2012 · 51:1368-1374

DOI 10.1007/s00120-012-3011-6

Online publiziert: 8. Oktober 2012

(c) Springer-Verlag Berlin Heidelberg 2012
M. Spahn $\cdot$ K. Haeni

Urologische Universitätsklinik, Inselspital Bern, Bern

Umgang mit dem Prostatakarzinom bei über 75-jährigen Männern

\section{Aktiv oder passiv?}

\begin{abstract}
Die Inzidenz maligner Erkrankungen in westlichen Industrienationen ist nicht zuletzt aufgrund der zunehmenden Lebenserwartung in den vergangengen Jahrzehnten dramatisch angestiegen. Annähernd $30 \%$ aller Patienten bei denen ein maligner Tumor diagnostiziert wird sind $>75$ Jahre. Auch das Prostatakarzinom (PCa), die am häufigsten diagnostizierte Krebserkrankung des Mannes und dritthäufigste zum Tode führende Tumorerkrankung in westlichen Industrienationen ist trotz des breiten Einsatzes des PSA-Screenings zur Früherkennung auch weiterhin eine Erkrankung des älteren Mannes [1, 2]. Bei Diagnosestellung beträgt das mediane Alter 68 Jahre und nahezu jeder 4. betroffene Mann ist >75 Jahre [3]. Etwa $71,2 \%$ der jährlich auftretenden $\mathrm{PCa}$-bedingten Todesfälle in den USA betreffen Männer dieser AItersgruppe [3].
\end{abstract}

Die epidemiologische Entwicklung mit zunehmender Alterung der Bevölkerung und die steigende Lebenserwartung lassen eine massive Zunahme der Prostatakarzinomfälle erwarten. Die aktuellen Leitlinien urologischer Fachgesellschaften geben keine spezifischen Therapieempfehlungen für ältere Patienten. Derzeit liegt lediglich ein Expertenkonsens der Internationalen Gesellschaft für Geriatrische Onkologie (SIOG) vor [4]. Obwohl prinzipiell Einigkeit darüber besteht, auf ein PSA-Screening bei Männern ab dem 75. Lebensjahr zu verzichten, hat die im Jahr 2011 veröffentlichte Empfehlung der amerikanischen „Preventive Service Task Force" (USPSTF) zum PSA-Screening (prostataspezifisches Antigen) zu kontroversen Diskussionen geführt [5].

Ein hohes Alter allein sollte weder die Tumordiagnostik noch die Behandlung eines Tumorleidens generell ausschließen. Vielmehr ist es erforderlich, die Risiken und Nutzen unterschiedlicher Behandlungsformen für jeden Patienten individuell einzuschätzen um Maßnahmen zu vermeiden, die die Lebensqualität eines Mannes beeinflussen ohne das Überleben relevant zu verlängern. Das Ziel dieses Artikels ist es, dem behandelnden Urologen eine Übersicht über den aktuellen Stand der Literatur zu geben, um die Diagnostik und Behandlung älterer Männer mit Prostatakarzinom besser einschätzen und steuern zu können.

\section{Lebenserwartung und Gesundheitsstatus}

Die Lebenserwartung eines Patienten wird als eine der wichtigsten Grundlagen für die Therapieentscheidung beim PCa angesehen. Vielfach wird allein das Alter eines Mannes als Grundlage für die Therapiewahl verwendet. Dieses Vorgehen lässt allerdings die große Varianz innerhalb einzelner Altersgruppen unberück- sichtigt. Ein 75-jähriger Mann hat z. B. eine durchschnittliche Lebenserwartung von 10,5 Jahren, gesunde Männer dieser Altersgruppe werden jedoch voraussichtlich noch 14,2 Jahre leben und jene mit schwerwiegenden Komorbiditäten nur 4,9 Jahre [6]. Demnach ist es erforderlich, weitere Faktoren in die Beurteilung der zu erwartenden Lebenserwartung mit einzubeziehen. Hierfür stehen unterschiedliche Möglichkeiten zur Verfügung.

Eine der wichtigsten Einflussgrößen sind die bestehenden Begleiterkrankungen. Insbesondere bei älteren Menschen spielen diese eine entscheidende Rolle. Bereits im Alter von 65 Jahren weist jeder 2. Mann mindestens eine chronische Erkrankung auf [7]. Diese Zahl steigt mit zunehmendem Lebensalter weiter an, so dass bei den $>75$-jährigen Männern in $>75 \%$ zwei oder mehr Komorbiditäten vorliegen. Der Charlson-Komorbiditätsindex wird vielfach für die Einschätzung der Schwere bestehender Begleiterkrankungen herangezogen und ist ein einfach $\mathrm{zu}$ verwendendes Instrument mit dem sich das allgemeine Sterblichkeitsrisiko effizient abschätzen lässt (• Tab. 1, [8]). Auch die Eigenständigkeit einer Person, sowie die kognitiven Fähigkeiten beeinflussen die Lebenserwartung älterer Menschen. Anhand existierender Fragebögen sind sowohl die Hilfsbedürftigkeit bei allgemeinen täglichen Aktivitäten (Körperpflege, Ankleiden, Mobilität, Nahrungsaufnahme u. a.) als auch die geistige Leis- 
Tab. 1 Der Charlson-Komorbiditätsindex berücksichtigt die Summe relevanter Begleiterkrankungen hinsichtlich der Lebenserwartung

\begin{tabular}{|c|c|c|}
\hline Komorbidität & Definition & $\begin{array}{l}\text { Bewer- } \\
\text { tung }\end{array}$ \\
\hline Herzinfarkt & $\begin{array}{l}\text { Pat. mit Hospitalisierung wegen elektrokardiographisch und/ } \\
\text { oder enzymatisch nachgewiesenem Herzinfarkt }\end{array}$ & 1 \\
\hline Herzinsuffizienz & $\begin{array}{l}\text { Pat. mit nächtlicher oder durch Anstrengung induzierte } \\
\text { Dyspnoe mit Besserung der Symptomatik unter Therapie }\end{array}$ & 1 \\
\hline $\begin{array}{l}\text { Periphere arterielle } \\
\text { Verschlusskrankheit }\end{array}$ & $\begin{array}{l}\text { Pat. mit Claudiacatio intermittens, peripherer Bypass, akutem } \\
\text { arteriellem Verschluss, abdominales oder thorakales } \\
\text { Aneurysma }\end{array}$ & 1 \\
\hline $\begin{array}{l}\text { Zerebrovaskuläre } \\
\text { Erkrankungen }\end{array}$ & Pat. mit TIA oder Apoplex ohne schwerwiegenden Residuen & 1 \\
\hline Demenz & Pat. mit chronischem kognitivem Defizit & 1 \\
\hline $\begin{array}{l}\text { Chronische Lungen- } \\
\text { erkrankung }\end{array}$ & $\begin{array}{l}\text { Pat. mit pulmonal bedingter Dyspnoe bei leichter oder } \\
\text { mäßig schwerer Belastung ohne Therapie oder Pt. } \\
\text { Mit anfallsweiser Dyspnoe (Asthma) }\end{array}$ & 1 \\
\hline Kollagenose & $\begin{array}{l}\text { Polymyalgia rheumatica, Lupus erythamatodes, schwere } \\
\text { rheumatoide Arthritis polymyositis }\end{array}$ & 1 \\
\hline Ulkuskrankheit & Pat. die bereits einmal wegen Ulzera behandelt wurden & 1 \\
\hline $\begin{array}{l}\text { Leichte Leber- } \\
\text { erkrankung }\end{array}$ & Leberzirrhose ohne portale Hypertonie & 1 \\
\hline $\begin{array}{l}\text { Diabetes mellitus (oh- } \\
\text { ne Endorganschäden) }\end{array}$ & Pat. mit DM und medikamentöser Therapie & 1 \\
\hline Hemiplegie & & 2 \\
\hline $\begin{array}{l}\text { Mäßig schwere } \\
\text { Nierenerkrankung }\end{array}$ & z. B. Dialysepflichtigkeit & 2 \\
\hline $\begin{array}{l}\text { Diabetes mellitus (mit } \\
\text { Endorganschäden) }\end{array}$ & $\begin{array}{l}\text { Oder zurückliegender Hospitalisationen wegen } \\
\text { hyperosmolarem Koma oder Ketoazidose }\end{array}$ & 2 \\
\hline Tumorerkrankung & $\begin{array}{l}\text { Sämtliche solide Tumore ohne Metastasennachweis } \\
\text { innerhalb der letzten } 5 \text { Jahre }\end{array}$ & 2 \\
\hline Leukämie & Akute und chronische Leukämien & 2 \\
\hline \multirow[t]{2}{*}{ Lymphom } & Multiples Myelom & \multirow[t]{2}{*}{2} \\
\hline & Hodgkin- und Non-Hodgkin-Lymphom & \\
\hline $\begin{array}{l}\text { Mäßig schwere Leber- } \\
\text { erkrankung }\end{array}$ & $\begin{array}{l}\text { Leberzirrhose mit portaler Hypertonie ohne stattgehabte } \\
\text { Blutung und Pat. mit Varizenblutung in der Anamnese }\end{array}$ & 3 \\
\hline $\begin{array}{l}\text { Metastasierter solider } \\
\text { Tumor }\end{array}$ & & 6 \\
\hline AIDS & & 6 \\
\hline
\end{tabular}

tungsfähigkeit bei alltäglichen Verrichtungen (Umgang mit Finanzen, Medikation, Nutzung öffentlicher Verkehrsmittel und Telekomunikationsmittel etc.) einfach beurteilbar $[9,10,11]$.

Zusätzlich wurde der Ernährungsstatus als Einflussgröße identifiziert. Unterernährung und ein größerer Gewichtsverlust in einem kurzen Zeitintervall sind im Alter mit einer höheren Mortalität verbunden [12].

Die Lebenserwartung hängt demnach nicht allein vom Alter, sondern von einer Reihe zusätzlicher Faktoren ab, die mit berücksichtigt werden müssen.

\section{Aktuelle Therapieempfehlungen}

Die oben genannten Kriterien bildeten die Grundlage für die im Jahr 2010 veröffentlichten Empfehlungen eines Expertengremiums der Internationalen Gesellschaft für Geriatrische Onkologie (SIOG) zur Behandlung des Prostatakarzinoms bei älteren Männern [4]. Für das lokal begrenzte Tumorstadium empfiehlt die SIOG für gesunde aktive Männer in gutem Ernährungszustand eine Standardtherapie in Analogie zu der bei jüngeren Männern. Für Patienten mit geringen Komorbiditäten oder leichter Hilfsbedürftigkeit bei täglichen Arbeiten wird eine Bestrah- lung und bei stärkeren Beeinträchtigungen die Hormontherapie angeraten. Lediglich für die Gruppe der schwerstkranken multimorbiden Männer im höheren Lebensalter wird eine palliative Therapie empfohlen. Diese Empfehlungen bedürfen einer kritischen Betrachtung.

Ziel einer jeglichen PCa-Therapie sollte es sein, mögliche durch die Erkrankung auftretende Einschränkungen der Lebensqualität zu vermeiden und das Leben der Patienten zu verlängern. Als Argumentationsgrundlage für eine aktive, aggressivere Therapie diente dem SIOG-Gremium eine retrospektive Analyse von 330 Männern im Alter von 70-74 Jahren deren PCa konservativ therapiert worden war. Die tumorbedingte Sterblichkeit dieser Männer war stark von der Tumordifferenzierung abhängig und lag zwischen $22 \%$ (Gleason-Score $<7$ ) und $67 \%$ (Gleason-Score 8-10, [13]). Unberücksichtigt blieb bei der Interpretation dieser Daten, dass die Diagnosestellung im Zeitraum von 1971-1984 (also in der Prä-PSAÄra) erfolgte. Inwiefern diese Tumore mit den heutigen Verhältnissen vergleichbar sind ist fraglich. Erstaunlicherweise blieb eine ähnliche Untersuchung aus dem Jahr 2009 unberücksichtigt [14]. In dieser wurden Patienten analysiert, deren PCa nach Einführung des PSA-Tests in den Jahren 1992-2002 diagnostiziert wurde. In der Altersgruppe der 75- bis 79-Jährigen starben deutlich weniger Männer am Prostatakarzinom als in der Prä-PSA Ära. Die 10-Jahres-PCa-Mortalität lag in dieser Untersuchung zwischen 15\% (GleasonScore $<7$ ) und 30\% (Gleason-Score 8-10).

Diese Ergebnisse weisen in eine ähnliche Richtung wie die der SPCG-4-Studie und die bisher vorliegenden Auswertungen der PIVOT-Studie („prostate cancer intervention versus observation trial“), die gezeigt haben, dass insgesamt nur wenige der Patienten am PCa versterben. Lediglich Männer mit „High-risk-Tumoren“ und/oder hohen PSA-Werten weisen eine höhere Tumorsterblichkeit auf [15]. Einige retrospektive Studien berichten darüber, dass insbesondere ältere Männer gehäuft derartige lokal fortgeschrittene „Highrisk-Tumore" aufweisen [16, 17, 18]. Diese Beobachtung lässt jedoch keinen Rückschluss zu, dass Männer dieser Altersgruppe wirklich ein höheres Risiko für die 


\section{Hier steht eine Anzeige.}

Springer 
Hier steht eine Anzeige.

黛 Springer 
Entwicklung aggressiverer PCa aufweisen. Inwiefern Selektionskriterien, die zur Diagnostik und Therapie geführt haben, diese Beobachtung beeinflusst haben bleibt unklar. Prospektive Studien, die die Verteilung unterschiedlicher Risikogruppen bei Männern $>75$ Jahre untersucht haben liegen derzeit nicht vor.

In einer Untersuchung der CaPSUREDatenbank („cancer of the prostate strategic urologic research endeavor") fanden Bechis et al. [18] in 26\% der älteren Männer ein „High-risk-PCa“ [definiert durch einen CAPRA-Score („,cancer of the prostate risk assessment") von 6-10].

Die Behandlung dieser Patientengruppe war uneinheitlich. Nur eine Minderheit wurde radikal prostatektomiert (3\%), die Mehrzahl erhielt entweder eine Bestrahlung (21\% externe Radiation, 14\% Brachytherapie), eine Hormontherapie (41\%) oder „watchful waiting“ (WW; 19\%). Der Vergleich der Behandlungsergebnisse der einzelnen Therapiemodalitäten zeigte eine 46\%ige Risikoreduktion der PCa-Mortalität in der „High-risk-Gruppe“ nach kurativer Therapie im Vergleich zu den konservativen Behandlungen. Interessanterweise fand sich kein Unterschied zwischen einer sofortigen Hormontherapie und WW. Obgleich eine Reduktion des Mortalitätsrisikos von $46 \%$ auf den ersten Blick ein beachtlicher Behandlungserfolg $\mathrm{zu}$ sein scheint ist diese Beobachtung zu relativieren. Insgesamt verstarben nur wenige Patienten überhaupt an ihrem PCa. Von den $392>75$-jährigen Männern mit „High-risk-Tumoren“ in der CaPSUREDatenbank verstarben nach einem Median von 5,3 Jahren insgesamt 183 Männer (47\%), aber lediglich 36 (20\%) der Todesfälle waren tumorbedingt. Die Sterblichkeit an konkurrierenden Ursachen war demnach 5fach höher $(\mathrm{n}=147)$.

Die vorliegenden Informationen sowohl aus retrospektiven, als auch aus den zwei prospektiven Studien (SPCG-4, PIVOT) zeigen eine insgesamt geringe $\mathrm{PCa}-$ Mortalität bei Männern bei denen die Diagnosestellung im höheren Alter erfolgte. Die Gruppe der „High-risk-PCa-Patienten“ wurde als die Subpopulation mit dem höchsten Sterblichkeitsrisiko identifiziert. Dennoch ist auch für diese Gruppe die Tumorsterblichkeit relativ gering und es überwiegt die nicht tumorbedingte

Urologe 2012 · 51:1368-1374 DOI 10.1007/s00120-012-3011-6

(c) Springer-Verlag Berlin Heidelberg 2012

\section{Spahn $\cdot$ K. Haeni \\ Umgang mit dem Prostatakarzinom bei über 75-jährigen Männern. Aktiv oder passiv?}

\section{Zusammenfassung}

Die steigende Lebenserwartung und die zunehmende Zahl älterer Menschen in der Bevölkerung westlicher Industrienationen rücken das Prostatakarzinom (PCa) im höheren Lebensalter zunehmend in den Fokus des Interesses. Einigkeit besteht darin, dass > 75-jährige Männer von einem PSA-Screening nicht profitieren. Ein höheres Alter allein sollte jedoch weder die Diagnostik noch die Behandlung eines Tumorleidens generell ausschließen. Zu berücksichtigen ist aber gerade beim Prostatakarzinom das Risiko von Überdiagnostik und Übertherapie. Die Mehrzahl der älteren Männer leidet an einer Vielzahl von Begleiterkrankungen, welche die Lebenserwartung einschränken, und das konkurrierende Mortalitätsrisiko übersteigt das der Tumorerkrankung um ein Vielfaches. Der behandelnde Urologe und der betroffene $\mathrm{Pa}$ tient sollten deshalb mögliche Auswirkungen einer Diagnosestellung und Therapie auf die Lebensqualität berücksichtigen.

Alter, bestehende Komorbiditäten und die individuelle kognitive und körperliche Leistungsfähigkeit stellen neben spezifischen Tumorparametern gute Kriterien für eine individualisierte Behandlungssteuerung dar.

Bei gesunden, aktiven >75-jährigen Männern mit „High-risk-PCa-Kriterien“ und Patienten mit einer PSA-Verdoppelungszeit $<12$ Monate sollte eine Therapie in Erwägung gezogen werden. Alle anderen Patienten in diesem Alter profitieren vermutlich nicht von einer Behandlung des PCa.

\section{Schlüsselwörter}

Prostatakarzinom · PSA-Screening .

Überdiagnostik · Übertherapie .

Begleiterkrankungen

\section{Approach to prostate cancer in men older than 75 years. Active or passive?}

\section{Abstract}

Based on the exponential aging of the population and the increasing life expectancy in industrialized western countries, prostate cancer ( $\mathrm{PCa}$ ) in elderly men is becoming a disease of increasing significance. Consensus exists that men over the age of 75 years should not be screened for PCa; however, higher age as a single parameter should not exclude men from being diagnosed with prostate cancer and treated accordingly. It is well-known that overdiagnosis and overtreatment are frequent in this age group. Competing mortality risks of men older than 75 years may supersede the risk of dying from PCa several fold. Both the treating physician and the patient himself should therefore balance the possible risks and benefits of diagnosing and treating prostate cancer concerning the impact on quality of life. This is of special importance when taking into ac- count that the complication rates of curative treatment modalities are higher in older patients than in younger men and that hormonal treatment might have negative effects especially in older men.

Age, existing comorbidities, cognitive and physical status in combination with specific tumor parameters are useful tools for an individualized treatment.

Therapy should be considered for healthy, active men aged 75 years or older who present with high-risk PCa and/or with a PSA doubling time $<12$ months. Elderly men who are unfit or have low to intermediate risk PCa will most likely not benefit from treatment.

\section{Keywords}

Prostate cancer . PSA screening .

Overdiagnosis - Overtreatment .

Comorbidities
Mortalität um ein Vielfaches. Bei der Betrachtung der Prostatakarzinomerkrankung ist es demnach aufgrund des langsamen Tumorwachstums insbesondere bei älteren Patienten von Bedeutung, das Gesamtüberleben getrennt vom tumorspezifischen Überleben zu betrachten.

\section{Prostatakarzinombedingte Mortalität und konkurrierende Sterblichkeit}

Der Einfluss sowohl von spezifischen Tumorparametern als auch von Alter und Komorbiditäten auf das Überleben kon- 
Tab. 2 10-Jahres-Gesamt- und prostatakarzinomspezifische Mortalität von >75-jährigen

Männern mit lokalisiertem Prostatakarzinom. (Mod. nach Albertsen et al. [19])

\begin{tabular}{|llllll}
\hline & & cT1c & cT2 & \\
\hline CCI & Gleason-Score & $5-7$ & $8-10$ & $5-7$ & $8-10$ \\
\hline 0 & Gesamtmortalität (\%) & 67,1 & 77,6 & 67,4 & 83,4 \\
\cline { 2 - 6 } & PCa-Mortalität (\%) & 14,0 & 27,5 & 14,2 & 27,9 \\
\hline \multirow{2}{*}{1} & Gesamtmortalität (\%) & 76,8 & 92,4 & 77,3 & 87,6 \\
\cline { 2 - 6 } & PCa-Mortalität (\%) & 9,1 & 23,7 & 13,3 & 19,6 \\
\hline \multirow{2}{*}{$>2$} & Gesamtmortalität (\%) & 74,4 & 93,4 & 88,9 & 88,8 \\
\cline { 2 - 7 } & PCa-Mortalität (\%) & 5 & 18,8 & 9,9 & 15,7 \\
\hline CCl Charlson-Komorbiditätsindex. & & & & \\
\hline
\end{tabular}

servativ therapierter PCa-Patienten wurde von Albertsen et al. [13, 19] anhand der SEER-Daten konservativ behandelter PCa-Patienten untersucht. Hierbei konnte nachgewiesen werden, dass die 10-JahresSterblichkeit für alle Komorbiditäts- und Tumorstadien in der Gruppe der > 75-Jährigen erheblich war [19]. Zudem war die überwiegende Mehrzahl der Todesfälle nicht tumorbedingt (• Tab. 2). Aufgrund dessen stellt sich die Frage, ob $>75$-jährige PCa-Patienten überhaupt therapiert werden sollten, und ob es eine Subpopulation gibt, die von einer sofortigen Behandlung profitiert.

Die Daten von Albertsen et al. [13, 19] konnten zeigen, dass lediglich gesunde Männer (Charlson-Komorbiditätsindex $=0$ ) mit einem undifferenzierten Tumor (Gleason-Score 8-10) eine substantiell höhere PCa-bedingte Mortalität aufwiesen. Dennoch ist das allgemeine Sterblichkeitsrisiko auch für diese gesunden $\mathrm{Pa}$ tienten hoch (5- und 10-Jahres-Mortalität: 41-50\% und 77-83\%) und das Risiko des nicht tumorbedingten Todes übersteigt jenes der Tumorsterblichkeit um ein Vielfaches: Ein gesunder Mann (Charlson-Komorbiditätsindex $=0$ ) mit einem GleasonScore-8-10-Tumor hat ein 2- bis 3fach höheres allgemeines 5- und 10-Jahres-Sterblichkeitsrisiko im Vergleich zur PCa-Mortalität. Wie bereits dargelegt, ist jedoch lediglich eine Minderheit der Männer in diese Komorbiditätsgruppe einzuordnen. Etwa $75 \%$ aller $>75$-jährigen weisen einen Charlson-Komorbiditätsindex von $\geq 2$ auf. Diese Männer haben eine 10-Jahres-Sterblichkeit von $\geq 90 \%$ und das Risiko an anderen Ursachen als dem PCa zu versterben ist um bis zu 14fach höher als das Tumorsterblichkeitsrisiko.
Diese Daten zeigen sehr eindrücklich, dass offensichtlich nur eine Minderheit der $>75$-Jährigen überhaupt eine Lebenszeit von $>10$ Jahren aufweist. Ein kurativer Therapieansatz scheidet demzufolge für die Mehrzahl der Patienten aus und es stellt sich die Frage, inwiefern eine antihormonelle Behandlung durchgeführt werden sollte. Diese Frage war Gegenstand der EORTC-Studie 30891, die den Einfluss einer sofortigen Androgendeprivation im Vergleich zur verzögerten Behandlung zum Zeitpunkt des systemischen Progresses hinsichtlich des Überlebens untersucht hat [20]. Eingeschlossen wurden Patienten mit neu diagnostiziertem T0-4 N0-2 M0-PCa, die entweder eine lokale Therapie abgelehnt hatten oder aufgrund einer eingeschränkten Lebenserwartung bzw. schwerwiegender Komorbiditäten nicht für eine kurative Therapie in Frage kamen. Das mediane Alter der Gesamtgruppe betrug 73 Jahre. Für die Gruppe der sofort hormontherapierten Patienten zeigte sich im Vergleich zu der Gruppe der verzögert behandelten ein geringfügiger aber signifikanter Vorteil im Gesamtüberleben, jedoch nicht für das symptomfreie- oder das tumorspezifische Überleben. Die mediane Zeit bis zur Einleitung einer verzögerten Hormontherapie betrug 7 Jahre. In dieser Gruppe verstarben 25,6\% der Patienten ohne jemals eine Hormontherapie benötigt zu haben. Subgruppenanalysen konnten zeigen, dass Patienten mit einem initialen PSA-Wert $>50 \mathrm{ng} / \mathrm{ml}$ und/oder einer PSA-Verdoppelungszeit $<12$ Monate eine höhere Tumorsterblichkeit aufwiesen und von einer sofortigen Hormontherapie profitierten [21].

Das allgemeine Mortalitätsrisiko >75-jähriger Männer ist insgesamt hoch und übersteigt das der tumorbedingten Sterblichkeit um ein Vielfaches. Lediglich gesunde Männer mit undifferenzierten Tumoren (Gleason-Score 8-10) und jene mit PSA-Werten $>50 \mathrm{ng} / \mathrm{ml}$ und/oder einer PSA-Verdoppelungszeit $<12$ Monate haben ein höheres $\mathrm{PCa}$-Mortalitätsrisiko. Bei diesen Patienten sollte eine PCaTherapie in Erwägung gezogen werden. Alle anderen Männer dieser Altersgruppe mit PCa profitieren von einer Behandlung vermutlich nicht.

\section{Zusammenfassung}

- Die steigende Lebenserwartung und die zunehmenden Zahl älterer Menschen in der Bevölkerung westlicher Industrienationen erfordern eine kritische Betrachtung der Prostatakarzinombehandlung bei älteren Männern. Insbesondere bei den >75-jährigen Männern besteht aufgrund der in etwa $75 \%$ der Fälle vorliegenden multiplen Begleiterkrankungen eine deutlich eingeschränkte Lebenserwartung. Demzufolge übersteigt das konkurrierende Mortalitätsrisiko eines > 75-jährigen Mannes das der Tumorerkrankung um ein Vielfaches. Der behandelnde Urologe und der betroffene Patient sollten deshalb mögliche Auswirkungen der Diagnostik und nachfolgender Behandlungen auf die Lebensqualität berücksichtigen. Die Komplikationsraten sowohl kurativer Behandlungsverfahren als auch die negativen Auswirkungen einer Hormontherapie sind im Alter als gravierender einzuschätzen [22].

- Alter, bestehende Komorbiditäten und die individuelle kognitive und körperliche Leistungsfähigkeit stellen neben individuellen Tumorparametern gute Kriterien für eine individualisierte Behandlungssteuerung dar.

- Bei gesunden, aktiven >75-jährigen Männern mit „High-risk-PCa“ (Gleason-Score 8-10 und/oder PSA >50 ng/ $\mathrm{ml}$ ) und/oder Patienten mit einer PSAVerdoppelungszeit $<12$ Monaten sollte eine Therapie in Erwägung gezogen werden, alle anderen Patienten profitieren vermutlich nicht von der Behandlung des PCa in diesem Alter. 


\section{Korrespondenzadresse}

\section{PD Dr. M. Spahn}

Urologische Universitätsklinik,

Inselspital Bern,

Anna Seiler-Haus,

CH-3010 Bern, Schweiz

martin.spahn@insel.ch

Interessenkonflikt. Der korrespondierende Autor gibt für sich und seinen Koautor an, dass kein Interessenkonflikt besteht.

\section{Literatur}

1. Ferlay J, Parkin DM, Steliarova-Foucher E (2010) Estimates of cancer incidence and mortality in Europe in 2008. Eur J Cancer 46:765-781

2. Ferlay J, Shin HR, Bray F et al (2010) Estimates of worldwide burden of cancer in 2008: GLOBOCAN 2008. Int J Cancer 127:2893-2917

3. Ries L et al (2008) SEER Cancer Statistics Review, 1975-2005. Bethesda, MD: National Cancer Institute. Available at: http://seercancergov/csr/19752005/2008

4. Droz JP, Balducci L, Bolla M et al (2010) Management of prostate cancer in older men: recommendations of a working group of the International Society of Geriatric Oncology. BJU Int 106:462-469

5. USPSTF (2012) US Preventive Service Task Force (USPSTF) recommendation on Screening for Prostate Cancer. http://www.uspreventiveservicestaskforceorg/uspstf/

6. Walter LC, Covinsky KE (2001) Cancer screening in elderly patients: a framework for individualized decision making. JAMA 285:2750-2756

7. Fuchs J, Busch M, Lnge C et al (2002) Gender-sensitive public health publications in Germany? Results of a review of literature. Bundesgesundheitsblatt Gesundheitsforschung Gesundheitsschutz 55:576-586

8. Charlson ME, Pompei $P$, Ales KL, MacKenzie CR (1987) A new method of classifying prognostic comorbidity in longitudinal studies: development and validation. J Chronic Dis 40:373-383

9. Rockwood K, Stadnyk K, MacKnight C et al (1999) A brief clinical instrument to classify frailty in elderly people. Lancet 353:205-206

10. Katz S, Ford AB, Moskowitz RW et al (1963) Studies of Illness in the Aged. The index of Adl: a standardized measure of biological and psychosocial function. JAMA 185:914-919

11. Lawton MP, Brody EM (1969) Assessment of older people: self-maintaining and instrumental activities of daily living. Gerontologist 9:179-186

12. Blanc-Bisson C, Fonck M, Rainfray M et al (2008) Undernutrition in elderly patients with cancer: target for diagnosis and intervention. Crit Rev Oncol Hematol 67:243-254

13. Albertsen PC, Hanley JA, Fine J (2005) 20-year outcomes following conservative management of clinically localized prostate cancer. JAMA 293:20952101

14. Lu-Yao GL, Albertsen PC, Moore DF et al (2009) Outcomes of localized prostate cancer following conservative management. JAMA 302:1202-1209

15. Bill-Axelson A, Holmberg L, Ruutu M et al (2011) Radical prostatectomy versus watchful waiting in early prostate cancer. N Engl J Med 364:1708-1717
16. Richstone L, Bianco FJ, Shah HH et al (2008) Radical prostatectomy in men aged (or(70 years: effect of age on upgrading, upstaging, and the accuracy of a preoperative nomogram. BJU Int 101:541-546

17. Manton KG, Vaupel JW (1995) Survival after the age of 80 in the United States, Sweden, France, England, and Japan. N Engl J Med 333:1232-1235

18. Bechis SK, Carroll PR, Cooperberg MR (2011) Impact of age at diagnosis on prostate cancer treatment and survival. J Clin Oncol 29:235-241

19. Albertsen PC, Moore DF, Shih W et al (2011) Impact of comorbidity on survival among men with localized prostate cancer. J Clin Oncol 29:1335-1341

20. Studer UE, Whelan P, Albrecht W et al (2006) Immediate or deferred androgen deprivation for patients with prostate cancer not suitable for local treatment with curative intent: European Organisation for Research and Treatment of Cancer (EORTC) Trial 30891. J Clin Oncol 24:1868-1876

21. Studer UE, Collette L, Whelan P et al (2008) Using PSA to guide timing of androgen deprivation in patients with T0-4 N0-2 M0 prostate cancer not suitable for local curative treatment (EORTC 30891). Eur Urol 53:941-949

22. Lowrance WT, Elkin EB, Yee DS et al (2012) Locally advanced prostate cancer: a population-based study of treatment patterns. BJU Int 109:1309-1314

\section{Start des Großprojekts „OR.Net - Sichere und dynamische Vernetzung in Operationssaal und Klinik"}

Gemeinsam mit insgesamt fast 50 Partnern aus ganz Deutschland wird die Universität zu Lübeck neue Konzepte zur Integration unterschiedlichster medizintechnischer Geräte in Operationssälen und Kliniken erforschen. Das Projekt startet mit 15 Millionen Euro Bundesforschungsgeldern. In modernen Operationssälen entsteht bei den Ärzten zunehmend der Wunsch nach der Vernetzung und Integration medizinischer Geräte, um die notwendigen Operationen effizienter und präziser ausführen zu können.

Dabei soll gleichzeitig die technische Komplexität sinken, so dass sich das Ärzteteam besser auf seine eigentliche Aufgabe konzentrieren kann. Von einigen Firmen werden hier bereits Komplettlösungen angeboten, die jedoch meist unter mangelnder Modularität, Flexibilität und Austauschbarkeit leiden.

Im Sinne einer technisch optimalen und gleichzeitig kostengünstigen Ausstattung eines OPs wäre es sinnvoll, wenn Krankenhäuser sich ihre Operationssäle aus unterschiedlichen Angeboten selbst zusammenstellen und trotzdem die Vorteile eines integrierten Gesamtsystems in Anspruch nehmen könnten. Dafür müssen jedoch nicht nur gemeinsame technische Kommunikationsmöglichkeiten geschaffen und standardisiert, sondern auch die rechtlichen Rahmenbedingungen des Medizinproduktegesetzes eingehalten werden. Mit dem jetzt geförderten Projekt OR.Net verfolgt der Bund das Ziel, die anfallenden Probleme zu lösen und dabei möglichst viele Unternehmen, Forschungseinrichtungen und Krankenhäuser miteinander zu vernetzen, um einen möglichst breiten Konsens zu ermöglichen. Das Projekt beginnt am 1.September 2012 und läuft über drei Jahre.

Quelle:Universität zu Lübeck, www.uni-luebeck.de 